\title{
SYNTACTIC VARIATION OF
}

MAKASSARESE, A MEMBER OF THE SOUTH SULAWESI GROUP OF LANGUAGE IN AUSTRONESIAN GREAT FAMILY, CLASSROOM LANGUAGE ASSESSMENT by Weda Sukardi

FILE

TIME SUBMITTED SUBMISSION ID
PROFESOR_5_PEOPLE.PDF (748.02K)

21-JAN-2019 02:41PM (UTC+0700) 1066537611
WORD COUNT

2954

CHARACTER COUNT 16475 
PEOPLE: International Journal of Social Sciences

ISSN 2454-5899

Sukardi Weda

Special Issue Volume 3 Issue 1, pp. 01 - 13

Date of Publication: $1^{\text {th }}$ January, 2017

DOI-https://dx.doi.org/10.20319/pijss.2017.31.0113

This paper can be cited as: Weda, S. (2017). Syntactic Variation of Makassarese, A Member of the South Sulawesi Group of Language in Austronesian Great Family, Classroom Language Assessment. PEOPLE: International Journal of Social Sciences, 3(1), 01-13

This work is licensed under the Creative Commons Attribution-NonCommercial 4.0 International License. To view a copy of this license, visit http://creativecommons.org/licenses/by-nc/4.0/ or send a letter to Creative Commons, PO Box 1866, Mountain View, CA 94042, USA.

\title{
SYNTACTIC VARIATION OF MAKASSARESE, A MEMBER OF THE SOUTH SULAWESI GROUP OF LANGUAGE IN AUSTRONESIAN GREAT FAMILY, CLASSROOM LANGUAGE ASSESSMENT
}

\author{
Sukardi Weda \\ Universitas Negeri Makassar, South Sulawesi, Indonesia \\ sukardi.weda@unm.ac.id
}

\begin{abstract}
Makassarese belongs to the member of the South Sulawesi group of the great family of Austronesian languages of Indonesia which is spoken by 2,130,000 (2000 census). Their homeland is South Sulawesi but many of them live in other provinces in Indonesia. This research aims to investigate sentence structure of Makassarese as a member of the Malayo-Polynesian branch of the Austronesian language family. The study employed qualitative approach in which the researcher asked the subjects to write down the equivalent of Indonesian language in Makassarese. The Indonesian sentences consisting of 15 sentences. The subjects were the students of Graduate Program who attended Morphosyntax subject. The research finding reveals that Makassarese has two basic sentence patterns in terms of its syntactic variation, they are SVO and VSO. In Makassarese, pronoun 'I' has two forms, independent pronoun 'Inakke' (I) and ergative pronoun ' $-k a$ ' (I). Makassarese speakers employ some sentence patterns and pronouns with various purposes and functions.
\end{abstract}

Keywords 
Syntactic variation, constituent structure, Makassarese

\section{Introduction}

Makassarese is one of the languages in Austronesian great family used by Makassar people who live in South Sulawesi. Makassar people live in the southern part of Sulawesi island, Makassar as the capital of South Sulawesi, Gowa, Takalar, and Jeneponto. Makassarese is also spoken by people who live in Maros, Pangkep, and Bantaeng.

The word "Makassar" is always used to explain the word which follows it, e.g Makassar people, Makassar land, and Makassar Harbour Cicy (Polinggomang, 2002). Polinggomang therefore add that Makassar people is one of ethnic groups who live in the southern and western part of Sulawesi island. Language and Makassar people or in some literatures is called Macassar, Macassarese, Makasar, Makassa, Makassaarsche, Makassarese, Mangasara, Manggasa, dan Mengkasara (Yamaguchi, 2010). The Makassar people live in some areas in South Sulawesi, Maros, Gowa, Galesong, Takalar, Topejawa, Laikang, Cikoang, Jeneponto, and Bangkala (Mattulada, 2011).

Makassar people together with Bugis people live in south peninsula of Sulawesi island, and they come from Toraja family (Mattulada, 2011). Mattulada adds that Makassar people has their own language (dialect) used in the cultural and civilized lives.

Nowadays, Makassarese is spoken by 2.130 .000 people (Census 2000). L2 users are 400.000 and many ethnic Chinese speak Makassar dialect as L1 (Lewis, et al, 2015).

\section{Research Method}

The subjects of this study were Makassar people who speak Makassarese well. The subjects of the study were students of Teaching English as a Foreign Language (TEFL) Graduate Program, Universitas Negeri Makassar who attended Morphosyntax subject taught by the researcher. Those subjects were from Gowa, Takalar, Jeneponto, and Makassar.

The study was conducted by distributing the list of sentences, consisting of 15 sentences in Indonesian language. The subjects were therefore asked to translate those Indonesian sentences into Makassarese. Those sentences contain a wide variety of syntactic variation. Those sentences are as follows. 


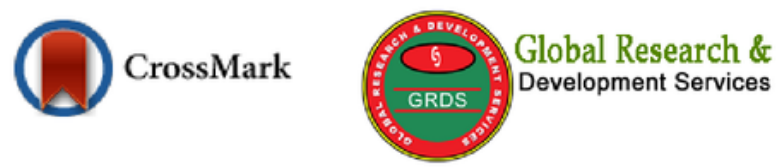

(1) Saya menulis surat untuk ibu di kampung. (I write a letter to my mother in the village).

(2) Ibu saya membeli ikan di pasar. (My mother buys fish in the market).

(3) Adik saya menangis setiap hari. (My younger brother cries every day).

(4) Kakak saya membantu bapak di kebun. (My elder brother helps father in the garden).

(5) SBY menonton pertandingan sepak bola di stadion Gelora Bung Karno. (SBY watches football competition in Gelora Bung Karno Stadion).

(6) Mahasiswa di perguruan tinggi sering tawuran (Students at the university often engage in a gang fight).

(7) Barack Obama berkunjung ke Indonesia beberapa bulan yang lalu. (Barack Obama visited Indonesia a few months ago).

(8) Banyak mahasiswa yang tidak belajar menjelang ujian semester. (Many students do not study before the examination).

(9) Pak Malik memiliki rumah besar dan indah. (Mr. Malik has large and beautiful house).

(10) Saya ingin makan apel asal Amerika. (I want to eat apple from USA).

(11) Saya ingin minum kopi pahit. (I want to drink bitter coffee).

(12) Nenek saya sedang sakit keras. (My grand father is sick).

(13) Padi di sawah telah menguning. (Rice in the rice field has become old).

The Makassarese sentences were analyzed to identify their constituent structure and sentence (construction) patterns. The aims of this analysis is to see and understand syntactic variation of Makassarese.

\section{Review of Literature}

Moravcsik (2006) argues that there are three main constituents of a sentence: subject, object, and verb - with six possible sentence structures. They are: Thai (SVO), Hindi (SOV), Tagalog (VSO), Malagasy (VSO), and two languages in Brasil, yakni bahasa Hixkaryana (OVS) dan Urubi (OSV). Other than these languages, Makassarese as a language spoken in South Sulawesi also has a wide variety of sentence pattern.

Makassarese is one of vernacular languages spoken by Makassar people who live in Sulawesi Selatan peninsula (Daeng, 2015). According to Kaseng (1978) in Daeng (2015) said 
that the areas of Makassarese language use are some parts of Pangkep, some parts of Maros, Makassar, Jeneponto, Bantaeng, Gowa, Takalar, some parts of Bulukumba, some parts of Sinjai, Selayar, and small parts of Bone.

Pelenkahu (1974) in Daeng (2015) has five dialects, those are: Lakiung, Turatea, Bantaeng, Konjo, and Selayar dialect.

In Makassarese, sentence is called lampangkana (Daeng, 2015). Sentence is bigger syntactic element than word, phrase, and clause (Daeng, 2015).

According to Daeng (2015), there are some types of sentences in Makassarese, those are simple sentences and complex sentences. Daeng therefore gave some simple sentences and complex sentences as follows.

a. Simple Sentence

1) Battuak ri Bantaeng subanngi.

'I came from Bantaeng yesterday.'

2) Annginungi kopi manggeku ri barikbasaka.

'My father drank coffee yesterday.'

3) Appilajarakak ammaca aksara lontarak.

'I am studying to learn aksara lontarak.'

b. Complex Sentence

1) Daeng Temba nipilei akjari kepala desa ri tumakbuttaya lanri carakdekna na bajik ri paranna tau.

'Daeng Temba was elected to be the head of village in the society because he is clever and respects to other people.'

2) Pattojeng-tojengi appilajaraka sollanna nucarakdek!

'Please study hard in other becoming smart person.'

\section{Findings and Discussion}

As a language used in every day life in some areas in South Sulawesi, Indonesia, Makassarese has some sentence patterns as revealed in the following sentences.

\section{Sentence (1)}

Makassarese, Takalar dialect

Nakke annulisi' sura' mange ri amma'ku ri kampong. 


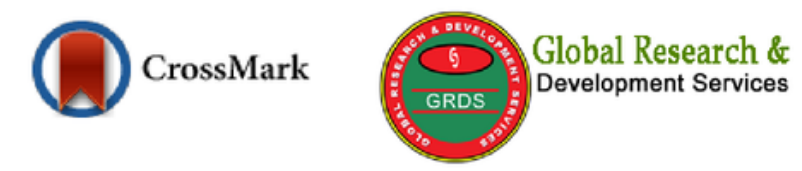

$$
\begin{array}{ccccc}
\text { S } & \text { V } & \text { DO } & \text { IO Adjunct } \\
\text { I } & \text { write } & \text { letter } & \text { mother-my in village }
\end{array}
$$

"I write a letter to my mother in the village."

Makassarese, Gow a dialect

Annulisi'ka' sura' mange ri ammakku ri kampong.

$$
\begin{array}{ccccc}
\text { V } & \text { S } & \text { DO } & \text { IO } & \begin{array}{c}
\text { Adjunct } \\
\text { write }
\end{array} \\
\text { I } & \text { letter } & \text { mother-my } & \text { village }
\end{array}
$$

"I write a letter to my mother in the village."

Makassarese, Jeneponto dialect

Annulisikka sura' mange ri ammakku ri kampong.

$$
\begin{array}{lllll}
\mathrm{V} & \mathrm{S} & \mathrm{DO} & \mathrm{IO} & \text { Adjunct }
\end{array}
$$

write I letter mother-my village

"I write a letter to my mother in the village."

Makassarese, Makassar dialect

Inakke annulisi surat mange ri ammakku ri kampong.

$\begin{array}{lllll}\mathrm{S} & \mathrm{V} & \mathrm{DO} & \text { IO }\end{array}$

I write a letter my mother village

"I write a letter to my mother in the village."

Sentence (2)

Makassarese, Takalar dialect

Amma'ku ammalli juku ri pasara.

$\begin{array}{ccc}\text { S } & \mathrm{O} & \text { Adjunct } \\ \text { mother-my buy fish } & \text { market }\end{array}$

"My mother buys fish in the market."

Makassarese, Gowa dialect

Amma'ku ammalli juku ri pasaraka.

$$
\text { S V O Adjunct }
$$

mother-my buy fish market

"My mother buys fish in the market."

Makassarese, Makassar dialect

Ammakku ammalli juku ri pasaraka.

$$
\mathrm{S} \quad \mathrm{V} \quad \mathrm{O} \text { Adjunct }
$$

mother-my buy fish market

"My mother buys fish in the market."

Makassarese, Jeneponto dialect

Ammakku ammalli juku ri pasaraka.

$$
\text { S } \quad \mathrm{V} \quad \mathrm{O} \text { Adjunct }
$$

mother-my buy fish market

"My mother buys fish in the market."

Kalimat (3) 
Makassarese, Takalar dialect

Andikku anggarru allo-allo.

$\mathrm{S}$ $\mathrm{V}$ Adjunct

younger brother-my cry every day

"My younger brother cries every day."

\section{Makassarese, Gowa dialect}

Andikku

anggarru allo - bangngi.

$\mathrm{S}$

$\mathrm{V}$

Adjunct

younger brother-my cry every day

"My younger brother cries every day."

Makassarese, Makassar dialect

Andikku

anggarru allo-allo.

$\mathrm{S}$

$\mathrm{V}$ Adjunct

younger brother-my cry every day

"My younger brother cries every day."

Makassarese, Jeneponto dialect

Andikku angngarru' allo-allona.

S V Adjunct

younger brother-my cry every day

"My younger brother cries every day."

Kalimat (4)

Makassarese, Takalar dialect

Kakangku ambantu bapakku ri koko.

S V O Adjunct

elder brother-my help father-my garden

"My elder brother helps my father in the garden."

Makassarese, Gowa dialect

Kakangku nabali mangekku ri kokonna.

$\begin{array}{llll}\mathrm{S} & \mathrm{V} & \mathrm{O} & \text { Adjunct }\end{array}$

elder brother-my help father-my garden

"My elder brother helps my father in the garden."

Makassarese, Makassar dialect

Daengku ambantui bapakku ri kokoa.
$\mathrm{V}$
$\mathrm{O}$ Adjunct

$\mathrm{S}$

elder brother-my help father-my garden 
"My elder brother helps my father in the garden."

Makassarese, Jeneponto dialect

Kakangku nabali tettaku ri kokoa.

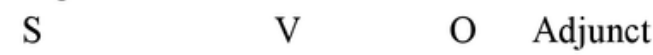

elder brother-my help father-my garden

"My elder brother helps my father in the garden."

\section{Sentence (5)}

Makassarese, Takalar dialect

SBY accini-cini pattandingang sepa' bola ri stadiung Bung Karno.

$$
\begin{array}{llll}
\mathrm{S} & \mathrm{V} & \mathrm{O} & \text { Adjunct }
\end{array}
$$

SBY watch football competition Bung Karno Stadion

"SBY watches football competition in Bung Karno Stadion."

\section{Makassarese, Gowa dialect}

SBY accini-ciniki lomba pagolo' ri stadion Gelora Bung Karno.

$$
\begin{array}{llll}
\mathrm{S} & \mathrm{V} & \mathrm{O} & \text { Adjunct }
\end{array}
$$

SBY watch football competition Bung Karno Stadion

"SBY watches football competition in Bung Karno Stadion."

\section{Makassarese, Makassar dialect}

SBY accinik pagolo ri stadion Gelora Bung Karno.

$$
\text { S V O } \quad \text { Adjunct }
$$

SBY watch football competition Bung Karno Stadion

"SBY watches football competition in Bung Karno Stadion."

Makassarese, Jeneponto dialect

SBY accini'-cini pagolo ri stadiong Gelora Bung Karno.

$$
\begin{array}{llll}
\mathrm{S} & \mathrm{V} & \mathrm{O} & \text { Adjunct }
\end{array}
$$

SBY watch football competition Bung Karno Stadion

"SBY watches football competition in Bung Karno Stadion."

\section{Sentence (6)}

Makassarese, Takalar dialect

Mahasiswa ri perguruang tinggia sangging la' sibajia.

$$
\mathrm{S}
$$

students
Mod V often engage in gang fight.

"Students at the university often engage in gang fight."

Makassarese, Gowa dialect 
Pakuliayya ri sikola tinggia sammaraki assibajjia.

$\mathrm{S}$ Adjunct Mod students university often engage in gang fight.

"Students at the university often engage in gang fight."

\section{Makassarese, Makassar dialect}

Mahasiswa tinggia sekolana biasa assibaji.

$$
\mathrm{S}
$$

Adjunct

Mod V

students university often engage in gang fight.

"Students at the university often engage in gang fight."

\section{Makassarese, Jeneponto dialect}

Mahasiswayya nangai assiba'jia.

$$
\mathrm{S}
$$$$
\text { Mod V }
$$

students often engage in gang fight.

"Students at the university often engage in gang fight."

\section{Sentence (7)}

\section{Bahasa Makassar, dialek Takalar}

Barack Obama menger ri Indonesia bulang-bulang allaloa.

$$
\text { S V Adjunct }
$$

Barack Obama visit Indonesia a few months ago

"Barack Obama visited Indonesia a few months ago."

\section{Makassarese, Gowa dialect}

Barack Obama battui ri Indonesia ri bulan laloa.

$$
\text { S V Adjunct }
$$

Barack Obama visit Indonesia a few months ago

"Barack Obama visited Indonesia a few months ago."

\section{Makassarese, Makassar dialect}

Barack Obama nabattui Indonesia ri bulan laloa.

$$
\begin{array}{lll}
\mathrm{S} & \mathrm{V} & \text { Adjunct }
\end{array}
$$

Barack Obama visit Indonesia a few months ago

"Barack Obama visited Indonesia a few months ago."

\section{Makassar, Jeneponto dialect}

Niaki Barack Obama mange ri Indonesia ri bulang-bulang laloa.

$$
\mathrm{V} \quad \mathrm{S} \quad \text { Adjunct }
$$

visit Barack Obama Indonesia a few months ago

"Barack Obama visited Indonesia a few months ago." 
Sentence (8)

Makassarese, Takalar dialect

Sanna mahasiswa tena na appilajara punna ero ujiang semester.

$\mathrm{S}$

$\mathrm{V}$

Adjunct

many students do not study before examination

"Many students do not study before examination."

Makassarese, Gow a dialect

Jai pakulia tena na appilajara na biringmo ulangang.

$$
\begin{array}{lll}
\mathrm{S} & \mathrm{V} & \text { Adjunct }
\end{array}
$$

many students do not study before examination

"Many students do not study before examination."

\section{Makassarese, Makassar dialect}

Jai mahasiswa tena appilajara punna laeroki ujian.

S V Adjunct

many students do not study before examination

"Many students do not study before examination."

\section{Makassarese, Jeneponto dialect}

Jai mahasiswa tena nappilajara punna eromi ujiang.

S V Adjunct

many students do not study before examination

"Many students do not study before examination."

\section{Sentence (9)}

Makassarese, Takalar dialect

Dg Malik nia balla lompona nampa ga'ya.

$$
\mathrm{S} \quad \mathrm{V}
$$

$\mathrm{O}$

Mr. Malik has big and beautiful house

"Mr. Malik has big and beautiful house."

\section{Makassarese, Gowa dialect}

Pak Malik ammallaki balla sanna lompona nampa bajiki kacinikanna.

\section{$\mathrm{S}$}

$$
\text { V }
$$

Mr. Malik has big and beautiful house

"Mr. Malik has big and beautiful house."

Makassarese, Makassar dialect

Bapak Malik napatai ballok lompoa era ga'ga. 


\section{$\begin{array}{lll}\mathrm{S} & \mathrm{V} & \mathrm{O}\end{array}$}

Mr. Malik has big and beautiful house

"Mr. Malik has big and beautiful house."

Makassarese, Jeneponto dialect

Nia' balla' lompo siangang ga' gana pa' Malik. $\mathrm{V}$

have big and beautiful house Mr. Malik

"Mr. Malik has big and beautiful house."

\section{Sentence (10)}

Makassarese, Takalar dialect

Eroka angnganre appele battu ri Amerika.

V1 S V2 O O Adjunct
want-I eat apple from USA
"I want to eat apple from USA."

"I want to eat apple from USA."

Makassarese, Gowa dialect

Eroka angnganre appele battu ri Amerika.

$$
\text { V1 S V2 O Adjunct }
$$

want-I eat apple from USA

"I want to eat apple from USA."

\section{Makassarese, Makassar dialect}

Eroka angnganre apel battua ri Amerika.

V1 S V2 O Adjunct

want-I eat apple from USA

"I want to eat apple from USA."

Makassarese, Jeneponto dialect

Erokka angnganre appele' battu ri Amerika.
V1 S V2
$\mathrm{O}$
Adjunct

want-I eat

apple from USA

"I want to eat apple from USA."

\section{Sentence (11)}

Makassarese, Takalar dialect

Asea ritanayya kunyi'mi.

$\mathrm{S}$ Adjunct $\mathrm{V}$

rice field rice have become old

"Rice in the field rice has become old." 
Makassarese, Gowa dialect

Asea ritanayya kunyi'mi.

$\mathrm{S}$ Adjunct $\mathrm{V}$

rice field rice have become old

"Rice in the field rice has become old."

Makassarese, Makassar dialect

Kunyimi asea ritanayya.

$\mathrm{V} \quad \mathrm{S}$ Adjunct

Have become old rice rice field

"Rice in the field rice has become old."

Makassarese, Jeneponto dialect

Didimi parea rigalunga.

$\mathrm{V} \quad \mathrm{S}$ Adjunct

Have become old rice rice field

"Rice in the field rice has become old."

\section{Sentence (12)}

Makassarese, Takalar dialect

Eroka angnginung kopi pai.'

$$
\text { V1-S V2 O }
$$

want-I drink bitter coffee

"I want to drink bitter coffee."

Makassarese, Gow a dialect

Eroka Angnginung kopi pai.

$$
\text { V1-S V2 O }
$$

want-I drink bitter coffee

"I want to drink bitter coffee."

Makassarese, Makassar dialect

Inakke ero angnginung kopi pai.

$\begin{array}{llll}\mathrm{S} & \mathrm{V} 1 & \mathrm{~V} 2 & \mathrm{O}\end{array}$

I want to drink bitter coffee

"I want to drink bitter coffee."

Makassarese, Jeneponto dialect

Erokka angnginung kopi pai'

V1-S V2 O 
want-I drink bitter coffee

"I want to drink bitter coffee."

Sentence (13)

Makassarese, Takalar dialect

Nenekku sannaki garringna

$\mathrm{S} \quad$ Adjective

grand mother-my sick

"My grand mother is sick."

Makassarese, Gowa dialect

Nenekku sannaki garringna.

S Adjective

grand mother-my sick

"My grand mother is sick."

Makassarese, Makassar dialect

Nenekku garring sarroi.

$\mathrm{S} \quad$ Adjective

grand mother-my sick

"My grand mother is sick."

Makassarese, Jeneponto dialect

Garring sannaki antokku.

Adjective $\mathrm{S}$

Sick grand mother-my

"My grand mother is sick."

\section{Concluding Remarks}

Makassarese as a vernacular language in South Sulawesi is widely spoken in everyday life. It belongs to the great family of Austonesian languages. The construction of Makassarese has two basic constituent structures or sentence patterns, they are SVO and VSO. In Makassarese, pronoun 'I' has two forms, independent pronoun 'Nakke or Inakke' (I), ergative pronoun '-ka' (I), as ini: Nakke annulisi' sura' mange ri amma'ku ri kampong (I write a letter to my mother in the village) and Annulisi'ka' sura' mange ri ammakku ri kampung (I write a letter to my mother in the village).

Makassarese speakers employ some sentence patterns and pronouns with various purposes and functions. One of the purposes is for pragmatic reasons, in which the Makassarese speakers use various expressions in morphosyntax (syntax and lexicon), they are mutually intelligible. 


\section{REFERENCES}

Daeng, Kembong. (2015). Makassarese Syntax. Makassar: Badan Penerbit UNM.

Lewis, M. Paul, Gary F. Simons, and Charles D. Fennig (eds.). (2015). Ethnologue: Languages of the World, Eighteenth edition. Dallas, Texas: SIL International. Online version: http://www.ethnologue.com/18/.

Mattulada. (2011). Menyusuri Jejak Kehadiran Makassar dalam Sejarah. Yogyakarta: Ombak.

Moravcsik, Edith. A. (2006). An Introduction to Syntax: Fundamentals of Syntactic Analysis. London: Continuum.

Yamaguchi, Masao. (2010). Tentang Nama Bahasa dan Dialek di Sulawesi Selatan dan Sekitarnya. Dalam Bahasa di Selat Makassar dan Samudera Pasifik (James T. Colin dan Chong Shin). Bangi: Institut Alam dan Tamadun Melayu (ATMA) UKM.

Weda. Sukardi. (2015). Saling Pengertian Dialek Bahasa Bugis. Dalam Konferensi Linguistik Tahunan (KOLITA) 13 Atmajaya Tingkat Internasional. Jakarta: Pusat Kajian Bahasa dan Budaya Unika Atmajaya. 
SYNTACTIC VARIATION OF MAKASSARESE, A MEMBER OF THE SOUTH SULAWESI GROUP OF LANGUAGE IN AUSTRONESIAN GREAT FAMILY, CLASSROOM LANGUAGE ASSESSMENT

ORIGINALITY REPORT

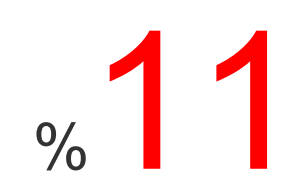

SIMILARITY INDEX
$\% 7$

INTERNET SOURCES
$\% 5$

PUBLICATIONS
$\% 11$

STUDENT PAPERS

PRIMARY SOURCES

1 Submitted to Shinas College of Technology Student Paper

2 www.grdspublishing.org Internet Source

EXCLUDE QUOTES ON

EXCLUDE

ON
EXCLUDE MATCHES

$<20$

WORDS

BIBLIOGRAPHY 